\title{
UNCOUNTABLY MANY INEQUIVALENT ANALYTIC ACTIONS OF A COMPACT GROUP ON $R^{n}$
}

\author{
R. S. PALAIS ${ }^{1}$ AND R. W. RICHARDSON, JR. ${ }^{2}$
}

1. Introduction. In this note we make use of recent results of McMillan [3] to construct examples which prove the following:

Theorem A. Let $G$ be a compact Lie group containing more than one element. For some positive integer $n$ there exists an uncountable family of real-analytic actions of $G$ on $n$-dimensional Euclidean space $R^{n}$ with nonhomeomorphic sets of stationary points.

Considering $S^{n}$ as the one point compactification of $R^{n}$ we obtain:

CoROLlaRY. There exists an uncountable family of continuous actions of $G$ on $S^{n}$ with nonhomeomorphic sets of stationary points.

According to a result of one of the authors [7] there are at most a countable number of inequivalent differentiable actions of a compact Lie group on a compact differentiable manifold. The results above show that both the compactness of the manifold and the differentiability of the action are necessary assumptions.

In the course of our proof we also prove the following theorem, which is an elementary consequence of the recent imbedding theorem of Grauert [2], but does not seem to have been explicitly stated elsewhere.

THEOREM B. Let $M_{1}$ and $M_{2}$ be second-countable real-analytic manifolds which are diffeomorphic. Then $M_{1}$ and $M_{2}$ are analytically diffeomorphic.

2. Preliminaries. If the group $G$ acts as a transformation group on the space $M$ we denote by $g \cdot x$ the image of the point $x \in M$ under the group element $g$. A point $x \in M$ is a stationary point if $g \cdot x=x$ for every $g \in G$. If $G$ is a Lie group and $M$ is a real-analytic manifold we say that the action of $G$ on $M$ is analytic if the map $(g, x) \rightarrow g \cdot x$ is an analytic map of $G \times M$ onto $M$.

The word differentiable as applied to manifolds, maps, etc., will always mean differentiable of class $C^{\infty}$.

3. The manifolds of McMillan. According to McMillan [3] there

Presented to the Society, February 22, 1962; received by the editors April 10, 1962.

Received by the editors April 10, 1962.

1 Partial support received from a National Science Foundation grant.

2 Partial support received from an Office of Naval Research contract. 
exists an uncountable family $\left(W_{\alpha}\right)$ of topologically distinct open 3manifolds such that, for each $\alpha$, the product $W_{\alpha} \times R^{m}$ is homeomorphic to $R^{m+3}$. It follows from the results of Moise [4], Cairns [1], and Whitney [10] that each $W_{\alpha}$ admits a real-analytic structure.

Stallings has recently shown [8] that if $M$ is a differentiable $n$ manifold $(n>4)$ which is homeomorphic to $R^{n}$, then $M$ is diffeomorphic to $R^{n}$. Let $W_{\alpha} \times R^{m}$ be given the induced analytic (and hence differentiable) structure as the product of the analytic manifolds $W_{\alpha}$ and $R^{m}$. It follows from Stallings' result that, for $m>1, W_{\alpha} \times R^{m}$ and $R^{m+3}$ are diffeomorphic. It will follow from Theorem $\mathrm{B}$ that they are analytically diffeomorphic.

4. Proof of Theorem B. The following theorem is due to Whitney $[9$, p. 86]:

4.1. Let $\epsilon$ be a positive continuous function on $R^{n}$ and $f$ a function of class $C^{k}$ on $R^{n}$. Then there exists an analytic function $g$ on $R^{n}$ such that, for every $x \in R^{n}$,

$$
|f(x)-g(x)|<\epsilon(x)
$$

and

$$
\left|\frac{\partial^{r} f}{\partial x_{i_{1}} \cdots x_{i_{r}}}(x)-\frac{\partial^{r} g}{\partial x_{i_{1}} \cdots x_{i_{r}}}(x)\right|<\epsilon(x)
$$

for $r \leqq k$ and $1 \leqq i_{1}, \cdots, i_{r} \leqq n$.

If $M$ is a closed analytic submanifold of $R^{n}$ with the induced Riemannian metric we obtain as an easy corollary:

4.2. Let $\epsilon$ be a positive continuous function on $M$ and $f$ a differentiable function on $M$. Then there exists an analytic function $g$ on $M$ such that, for every $x \in M$,

(a) $|f(x)-g(x)|<\epsilon(x)$ and

(b) $\|\operatorname{grad} f(x)-\operatorname{grad} g(x)\|<\epsilon(x)$.

If $\epsilon$ is a positive continuous function on $M$ and $f$ and $g$ are differentiable functions on $M$ satisfying (a) and (b) of 4.2 we say that $f$ is an $\epsilon$-approximation to $g$. If $f=\left(f_{1}, \cdots, f_{m}\right)$ and $g=\left(g_{1}, \cdots, g_{m}\right)$ are differentiable mappings of $M$ into $R^{m}$, we say that $f$ is an $\epsilon$ approximation to $g$ if, for each $i, f_{i}$ is an $\epsilon$-approximation to $g_{i}$.

We need the following lemma:

4.3. Lemma. Let $M_{1}$ and $M_{2}$ be complete Riemannian manifolds and let $f$ be a diffeomorphism of $M_{1}$ onto $M_{2}$. Then there is a positive continuous function $\epsilon$ on $M_{1}$ such that, if $g$ is a nonsingular map of $M_{1}$ into 
$M_{2}$ satisfying $\rho(f(x), g(x))<\epsilon(x)$ for all $x \in M_{1}$, then $g$ is a diffeomorphism of $M_{1}$ onto $M_{2}$.

Proof. It is no loss of generality to assume that $M_{1}=M_{2}=M$ and that $f$ is the identity map. We may also assume that $M$ is connected since if $\epsilon$ is small enough, $g$ will map each component of $M$ into itself. We next note that if $\rho(g(x), x)$ is even bounded, then $g$ is a proper map. This follows from the fact that on a complete Riemannian manifold a subset is relatively compact if and only if it is bounded. By [6, Theorem 4.2] $g$ is a covering map. In particular, $g$ maps $M$ onto $M$. It remains to prove that $g$ is one-to-one. We choose the bounded positive function $\epsilon$ on $M$ such that each point $x \in M$ has as neighborhood a geodesic ball of radius $2 \epsilon(x)$. If $\rho(g(x), x)<\epsilon(x)$ and $g(y)=g(z)=x$, then $y$ and $z$ both lie in the ball $B$ about $x$ of radius $\epsilon(x)$. Moreover $g$ maps $B$ into the ball $B^{\prime}$ about $x$ of radius $2 \epsilon(x)$. Since $B^{\prime}$ is simply connected and $g$ is a covering map, $g$ is one-to-one on each component of $g^{-1}\left(B^{\prime}\right)$. In particular $g$ is one-to-one on $B$ and thus $y=z$.

We return to the proof of Theorem B. According to [2] $M_{1}$ and $M_{2}$ may be analytically imbedded as closed submanifolds of (respectively) $R^{m}$ and $R^{q}$; we shall identify $M_{1}$ and $M_{2}$ with their images in $R^{m}$ and $R^{q}$. Let $f$ be a diffeomorphism of $M_{1}$ onto $M_{2}\left(\subset R^{q}\right)$. It is well known that there exists a positive continuous function $\eta$ on $M_{2}$ with the following property: If $U$ is the set of pairs $(x, y) \in\left(M_{2} \times R^{q}\right)$ such that $y$ is orthogonal to the tangent space of $M_{2}$ at $x$ and $\|y\|<\eta(x)$, then $(x, y) \rightarrow x+y$ is a homeomorphism of $U$ onto an open "tubular" neighborhood $N\left(M_{2}\right)$ of $M_{2}$ in $R^{q}$. Moreover, if $z \in R^{q}, x \in M_{2}$, and $\|z-x\|<\eta(x)$, then $z \in N\left(M_{2}\right)$. Define $p: N\left(M_{2}\right) \rightarrow M_{2}$ by $p(x+y)=x$ for $(x, y) \in U$. Then $p$ is an analytic retraction of $N\left(M_{2}\right)$ onto $M_{2}$. Let $\eta_{1}=q^{-1 / 2}(\eta \circ f)$. If $g: M_{1} \rightarrow R^{q}$ is an $\eta_{1}$ approximation to $f$, then $p \circ g$ is well defined. By 4.2 and 4.3 it will suffice to show that if $\epsilon$ is sufficiently small and $g$ is an $\epsilon$-approximation to $f$, then $p \circ g$ is nonsingular. Since $p \circ f=f$ is nonsingular this is clear.

5. Construction of the actions. In this section we identify the analytic manifolds $W_{\alpha} \times R^{m}$ and $R^{m+3}$ by means of an analytic diffeomorphism.

Let $G$ be a nontrivial compact Lie group. Let the integer $m(>1)$ be chosen such that there is a faithful representation $g \rightarrow T_{g}$ of $G$ in $\mathrm{GL}(m, R)$ which, considered as an action of $G$ on $R^{m}$, has only the origin $O$ as stationary point. It follows from standard theorems that the action thus obtained of $G$ on $R^{m}$ is real-analytic. For each $\alpha$ we define an action of $G$ on $W_{\alpha} \times R^{m}\left(=R^{m+3}\right)$ by $g \cdot(w, x)=\left(w, T_{0}(x)\right)$. 
This action is real-analytic and the set of stationary points is precisely $W_{\alpha} \times\{0\}$. Since the manifolds $W_{\alpha}$ are nonhomeomorphic this completes the proof of Theorem A.

To prove the corollary we consider $S^{n}$ as the one-point compactification of $R^{n}(n=m+3)$; writing $S^{n}=R^{n} \cup\left\{p_{\infty}\right\}$ we extend each action of $G$ on $R^{n}$ to an action of $G$ on $S^{n}$ by setting $g \cdot p_{\infty}=p_{\infty}$. For each $\alpha$ the set of stationary points for the given action of $G$ on $S^{n}$ is homeomorphic to the one-point compactification of $W_{\alpha}$. Since these sets are nonhomeomorphic the corollary follows.

6. Dimension of orbits. If $G$ is one of the groups which can act transitively on spheres and the integer $n$ in Theorem $\mathrm{A}$ is properly chosen, for each action in the family the principal orbits will be of dimension $(n-4)$. We do not know if similar examples can be constructed in which there exist orbits of dimension $(n-3)$. It follows from the results of [5] that no such examples exist with $(n-2)$ dimensional orbits.

\section{REFERENCES}

1. S. S. Cairns, The triangulation problem and its role in analysis, Bull. Amer. Math. Soc. 52 (1946), 545-571.

2. H. Grauert, On Levi's problem and the imbedding of real-analytic manifolds, Ann. of Math. (2) 68 (1958), 460-472.

3. D. R. McMillan, Some contractible open 3-manifolds, Trans. Amer. Math. Soc. 102 (1962), 373-382.

4. E. E. Moise, Affine structures in 3-manifolds, Ann. of Math. (2) 56 (1952), 96114.

5. D. Montgomery, H. Samelson and C. T. Yang, Groups on $E^{n}$ with $(n-2)$ dimensional orbits, Proc. Amer. Math. Soc. 7 (1956), 719-728.

6. R. S. Palais, Natural operations on differential forms, Trans. Amer. Math. Soc. 92 (1959), 125-141.

7. - Equivalence of nearby differentiable actions of a compact group, Bull. Amer. Math. Soc. 67 (1961), 362-364.

8. J. Stallings, The piecewise-linear structure of Euclidean space, Proc. Cambridge Philos. Soc. 58 (1962), 481-489.

9. H. Whitney, Analytic extensions of differentiable functions defined in closed sets, Trans. Amer. Math. Soc. 36 (1934), 63-89.

10. —_, Differentiable manifolds, Ann. of Math. (2) 37 (1936), 645-680.

BRANDEIS UNIVERSITY AND UNIVERSITY OF WASHINGTON 